\title{
Implications of CoGeNT and DAMA for light WIMP dark matter
}

\author{
A. Liam Fitzpatrick, ${ }^{1}$ Dan Hooper, ${ }^{2,3}$ and Kathryn M. Zurek ${ }^{4}$ \\ ${ }^{1}$ Department of Physics, Boston University, Boston, Massachusetts 02215, USA \\ ${ }^{2}$ Particle Astrophysics, Fermi National Accelerator Laboratory, Batavia, Illinois 60563, USA \\ ${ }^{3}$ Department of Astronomy and Astrophysics, University of Chicago, Chicago, Illinois 60637, USA \\ ${ }^{4}$ Michigan Center for Theoretical Physics and Department of Physics, University of Michigan, Ann Arbor, Michigan 48109, USA
}

(Received 7 April 2010; published 11 June 2010)

In this paper, we study the recent excess of low energy events observed by the CoGeNT Collaboration, and discuss the possibility that these events originate from the elastic scattering of a light $\left(m_{\mathrm{DM}} \sim\right.$ 5-10 GeV) weakly interacting massive particle (WIMP). We find that such a dark matter candidate may also be capable of generating the annual modulation reported by DAMA, without conflicting with the null results from other experiments, such as XENON10. The regions implied by CoGeNT and DAMA are also near those required to produce the two observed CDMS events. A dark matter interpretation of the CoGeNT and DAMA observations favors a region of parameter space that is especially attractive within the context of asymmetric dark matter models. In such models, the cosmological dark matter density arises from the baryon asymmetry of the Universe, naturally leading to the expectation that $m_{\mathrm{DM}} \sim$ 1-10 GeV. We also discuss neutralino dark matter from extended supersymmetric frameworks, such as the next-to-minimal supersymmetric standard model. Lastly, we explore the implications of such a dark matter candidate for indirect searches, and find very encouraging prospects for experiments attempting to detect neutrino or gamma ray annihilation products.

DOI: 10.1103/PhysRevD.81.115005

PACS numbers: $95.35 .+\mathrm{d}$

\section{INTRODUCTION}

The direct and indirect detection of dark matter has recently become a topic of intense discussion and activity, with a number of reported signals having been interpreted as possible detections of particle dark matter. Among direct detection experiments, the DAMA Collaboration, which has collected over 1.17 ton yr of data, reports an annual modulation in their event rate with $8.9 \sigma$ significance, which they interpret as evidence of dark matter [1]. The CDMS experiment has also observed a small excess (2 events, corresponding to less than $2 \sigma$ significance) in their low energy nuclear recoil window [2]. Furthermore, the CoGeNT Collaboration has very recently announced the observation of an excess of events at low energies [3]. If the CoGeNT excess is interpreted as a detection of dark matter, this points to dark matter with a mass in the range of approximately $m_{\mathrm{DM}} \sim 5-10 \mathrm{GeV}$, and an elastic scattering cross section with nucleons of approximately $\sigma \sim 7 \times$ $10^{-41} \mathrm{~cm}^{2}$. Remarkably, a dark matter candidate with this approximate mass and cross section would also be capable of producing both events observed by CDMS, and the modulation signal reported by DAMA.

Indirect detection efforts have also recently produced a number of tantalizing, if unconfirmed, signals of dark matter. The PAMELA experiment has reported an excess of cosmic ray positrons between 10 and $100 \mathrm{GeV}$ relative to the predictions of standard cosmic ray models [4]. The Fermi Gamma Ray Space Telescope (FGST) Collaboration has also reported a significantly harder spectrum of electrons (plus positrons) than had been anticipated [5]. In addition, there are apparent excesses in radio and gamma rays originating from the inner kiloparsecs of the Milky Way: the WMAP haze [6], and the recently reported Fermi (gamma ray) haze [7]. Lastly, it has recently been shown that the spectrum and angular distribution of gamma rays from the Galactic center, as observed by the FGST, is consistent with an annihilating dark matter interpretation [8]. Although each of these observations is consistent with annihilating dark matter, it is not yet possible to rule out less exotic astrophysical origins, such as pulsars [9].

In this paper, we focus on recent results from direct detection experiments, and examine the implications of the detections made by CoGeNT, DAMA, and CDMS. ${ }^{1}$ We begin by discussing the compatibility of these observations with each other, and with the null results of other experiments, in particular, the recent low-threshold analysis of the XENON10 Collaboration [11]. We then turn to a discussion of dark matter models that are naturally able to accommodate the observations of CoGeNT, DAMA, and CDMS. Particularly attractive within this context are asymmetric dark matter (ADM) models [12,13], in which the dark matter abundance is related to the baryon asymmetry of the Universe. In such models, the dark matter's mass is predicted to be a factor of several times heavier than the proton, precisely within the region indicted by CoGeNT. We also explore supersymmetric models in which these

\footnotetext{
${ }^{1}$ The dark matter candidates giving rise to direct detection signals considered here are not the same as could generate the positron excesses in Fermi and PAMELA, though multiple components of dark matter could simultaneously generate multiple signals of different type in direct and indirect detection experiments [10].
} 
signals can be produced. In particular, we discuss neutralinos within the context of the next-to-minimal supersymmetric standard model (NMSSM). Lastly, we turn our attention to the prospects for indirect dark matter searches. We find that, while the region implied by CoGeNT is not ruled out by any indirect detection experiments, the outlook for dark matter's indirect detection is very encouraging in this scenario. In particular, neutrino and gamma ray searches for dark matter annihilation products are currently within a factor of $\sim 2$ of the sensitivity required to test many of the models capable of generating the signals reported by CoGeNT, DAMA, and CDMS.

\section{COGENT, DAMA, CDMS, AND XENON}

In this section, we explore whether a dark matter interpretation of the CoGeNT excess is consistent with the signals reported by DAMA and CDMS, and whether such an interpretation is consistent with the null results of XENON10 [14] and the CDMS silicon analysis [15,16]. In particular, we follow Refs. [17-20] in studying direct detection signals in the low mass weakly interacting massive particle (WIMP) region, including the possible effects of channeling in the DAMA experiment [21].

\section{A. Detection rates}

The differential rate of dark matter elastic scattering events per unit detector mass in nuclear recoil energy is given by

$$
\frac{d R}{d E_{R}}=N_{T} \frac{\rho_{\mathrm{DM}}}{m_{\mathrm{DM}}} \int_{|\vec{v}|>v_{\min }} d^{3} v v f\left(\vec{v}, \vec{v}_{e}\right) \frac{d \sigma}{d E_{R}},
$$

where $N_{T}$ is the number of target nuclei per unit mass, $m_{\mathrm{DM}}$ is the dark matter particle mass, $\rho_{\mathrm{DM}}=0.3 \mathrm{GeV} / \mathrm{cm}^{3}$ is the local dark matter density, $\vec{v}$ is the dark matter velocity in the frame of the Earth, $\vec{v}_{e}$ is the velocity of the Earth with respect to the galactic halo, and $f\left(\vec{v}, \vec{v}_{e}\right)$ is the distribution function of dark matter particle velocities, which we take to be a standard Maxwell-Boltzmann distribution:

$$
f\left(\vec{v}, \vec{v}_{e}\right)=\frac{1}{\left(\pi v_{0}^{2}\right)^{3 / 2}} e^{-\left(\vec{v}+\vec{v}_{e}\right)^{2} / v_{0}^{2}}
$$

The Earth's speed relative to the galactic halo is $v_{e}=$ $v_{\odot}+v_{\text {orb }} \cos \gamma \cos \left[\omega\left(t-t_{0}\right)\right]$ with $v_{\odot}=v_{0}+12 \mathrm{~km} / \mathrm{s}$, $v_{\text {orb }}=30 \mathrm{~km} / \mathrm{s}, \cos \gamma=0.51, t_{0}=$ June 2 nd, and $\omega=$ $2 \pi /$ yr. The parameter $v_{0}$ is expected to be roughly $v_{\text {rot }}$ [22], and we will consider $v_{0}=220 \mathrm{~km} / \mathrm{s}$ and $v_{0}=$ $270 \mathrm{~km} / \mathrm{s}$ in analysis as representative values. The upper limit of the velocity integration of Eq. (1) is the galactic escape velocity, $490 \mathrm{~km} / \mathrm{s} \leq v_{\text {esc }} \leq 730 \mathrm{~km} / \mathrm{s}$ at 90\% C.L. [23], and the minimum dark matter velocity, $v_{\min }$, is

$$
v_{\min }=\sqrt{\frac{E_{R} m_{N}}{2 \mu_{1}^{2}}}
$$

where $E_{R}$ is the recoil energy, $m_{N}$ is the mass of the target nucleus, and $\mu_{1}$ is the dark matter-nucleus reduced mass. For a spin-independent cross section between dark matter particles and nuclei, we have [24]

$$
\frac{d \sigma}{d E_{R}}=\frac{m_{N}}{2 v^{2}} \frac{\sigma_{N}}{\mu_{n}^{2}} \frac{\left[f_{p} Z+f_{n}(A-Z)\right]^{2}}{f_{n}^{2}} F^{2}\left(E_{R}\right),
$$

where $\mu_{n}$ is the reduced mass of the dark matter particle and nucleon (proton or neutron), $\sigma_{N}$ is the scattering cross section of the dark matter particle with neutrons, $Z$ and $A$ are the proton and atomic numbers of the nucleus, and $f_{n, p}$ are the coupling strengths of the dark matter particle to neutrons and protons, respectively. The couplings $f_{n, p}$ are calculated from a coherent sum over the couplings to the quark constituents of the nucleon. We use the WoodsSaxon form factor, $F\left(E_{R}\right)[25,26]$.

CoGeNT is an ultralow noise detector operating in the Soudan mine, consisting of $0.33 \mathrm{~kg}$ (fiducial mass) of germanium. Over a period of 56 days, the CoGeNT detector recorded approximately 100 events (with characteristics corresponding to interactions in the bulk of the crystal) above their expected background with ionization energy between $3.2 \mathrm{keVee}^{2}$ and their threshold of $0.4 \mathrm{keVee}$ [3]. The CoGeNT Collaboration has pointed out that their events can be fit very well by a $\sim 10 \mathrm{GeV}$ dark matter particle with an elastic scattering cross section of $\sim 7 \times$ $10^{41} \mathrm{~cm}^{2}$. In Fig. 1, we confirm this conclusion, where we show the parameter space region in which elastically scattering dark matter can accommodate the CoGeNT excess at $90 \%$ confidence. We have used $v_{0}=220 \mathrm{~km} / \mathrm{s}$ and $v_{\text {esc }}=$ $500 \mathrm{~km} / \mathrm{s}$ in the left panel, and $v_{0}=270 \mathrm{~km} / \mathrm{s}$ and $v_{\text {esc }}=$ $490 \mathrm{~km} / \mathrm{s}$ in the right. The constraints from XENON10 appear to rule out the DAMA favored region. However, there are significant sources of uncertainty in such constraints, and the allowed regions of parameter space are very sensitive to the assumptions one makes about the analysis and various experimental parameters. In Fig. 2, we show the parameter space region with more optimistic assumptions, the effects of which will be discussed in much more detail in the following sections. Here, and throughout this paper, $90 \%$ (99\%) confidence regions are defined as contours of $\chi^{2}=\chi_{\min }^{2}+4.61$ (9.21), while constraints from null experiments are defined as $90 \%$ limits based on the maximum gap [27] method. To carry out this fit, we have assumed that the background is well described by an exponential plus constant, and we have required bin by bin that the background not exceed the amplitude of the

\footnotetext{
${ }^{2}$ Ionization energies are given in units of keVee, or $\mathrm{keV}$ electron equivalent. For a $1 \mathrm{keV}$ nuclear recoil, for example, the equivalent electron energy (in keVee) is $q_{x} \times 1 \mathrm{keV}$, where $q_{x}$ is the quenching factor for the nuclear material composing the scintillator. For the germanium target used in CoGeNT, the quenching factor is approximately 0.2 to 0.3 for events in the energy range of the observed excess. For the materials composing the DAMA detectors, $q_{N a} \approx 0.3$ and $q_{I} \approx 0.09$.
} 

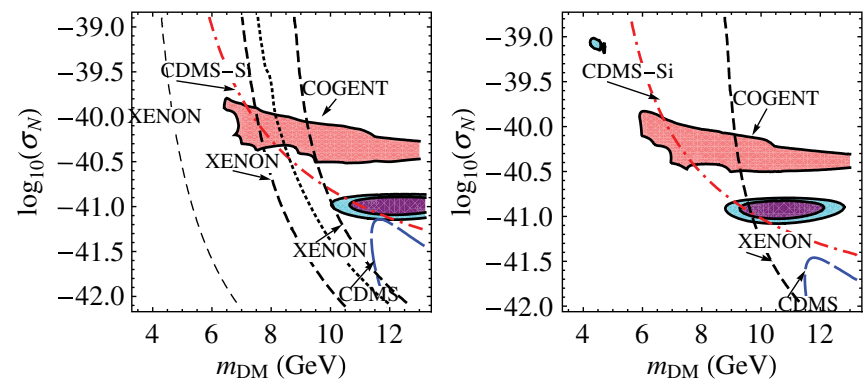

FIG. 1 (color online). Left panel: To illustrate the effect of uncertainties in the XENON10 scintillation factor, $L_{\text {eff }}$, we show the CoGeNT and DAMA allowed regions alongside constraints from the new XENON10 analysis using $L_{\text {eff }}=0.19$ (light gray dashed curve), and $L_{\text {eff }}$ at the central and lower $1 \sigma$ values from the new measurement [33] (two thick black dashed curves). In between the central and lower $1 \sigma L_{\text {eff }}$ curves we have taken $-\frac{1}{2} \sigma L_{\mathrm{eff}}$ values in only the three energy bins near XENON10's threshold (black dotted curve). We also show constraints from the CDMS silicon analysis (red dot-dashed curve), and the region in which the two events observed by CDMS can be produced (blue long dashed curve). Here, we have used $v_{0}=$ $220 \mathrm{~km} / \mathrm{s}$ and $v_{\text {esc }}=500 \mathrm{~km} / \mathrm{s}$. With the lower values of $L_{\text {eff }}$ values, the tension between XENON10 and CoGeNT is alleviated. Right panel: To illustrate the effect from changing the halo model, CoGeNT, CDMS, XENON10, and DAMA results are shown, but with $v_{0}=270 \mathrm{~km} / \mathrm{s}$ and $v_{\text {esc }}=490 \mathrm{~km} / \mathrm{s}$.
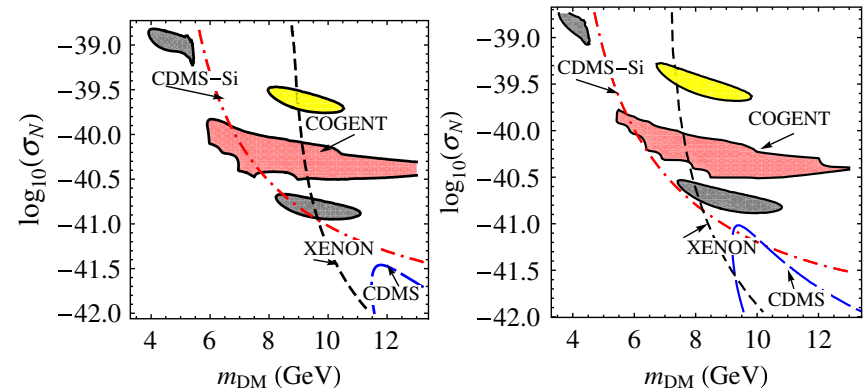

FIG. 2 (color online). The regions in the elastic scattering cross section (per nucleon), mass plane in which dark matter provides a good fit to the CoGeNT excess, compared to the region that can generate the annual modulation reported by DAMA at $90 \%$ confidence (darker gray regions). In this figure, we have adopted $v_{0}=270 \mathrm{~km} / \mathrm{s}$ and use two values of the galactic escape velocity: $v_{\text {esc }}=490 \mathrm{~km} / \mathrm{s}$ (left panel) and $v_{\text {esc }}=650 \mathrm{~km} / \mathrm{s}$ (right panel). In calculating the DAMA region, we have neglected the lowest energy bin (the effect of this is shown in later figures) and treated channeling as described in Ref. [28]. If a smaller fraction of events are channeled in DAMA than is estimated in Ref. [28], the DAMA region will move upward, toward the yellow regions (near $\sigma_{N} \approx 10^{-39.5} \mathrm{~cm}^{2}$, which include no effects of channeling), improving its agreement with CoGeNT. Also shown is the $90 \%$ C.L. region in which the 2 events observed by CDMS can be produced. If the escape velocity of the Galaxy is taken to be relatively large, this region can also approach those implied by CoGeNT and DAMA. Constraints from the null results of XENON10 and the CDMS silicon analysis are also shown. For the XENON10 constraint, we have used the lower estimate of the scintillation efficiency (at $1 \sigma)$ as described in Ref. [33]. dark matter signal. Without a constraint on dark matter signal to background, the entire spectrum is well fit by a pure exponential background. Tighter constraints on the amplitude of the background will correspond to the dark matter signal region shifting to larger cross sections. We fit the data in $0.05 \mathrm{keVee}$ bins from threshold at 0.4 to 1.8 keVee where the dark matter signal is negligible. Peaks in the data (consistent with a background from radioactive tin) at 1.1 and 1.29 keVee are fit by Gaussians of relative height 0.4 and with width consistent with the experimental resolution at those energies $(0.0774$ and $0.078 \mathrm{keVee}$, respectively). We can see that for appropriate choices of the halo model and the fraction of channeled events in DAMA, the CoGeNT region can be consistent at $90 \%$ C.L. with the DAMA signal and the null results XENON and CDMS-Si. Some consistency between the preferred region for CDMS with DAMA and CoGeNT can also be found. We now turn to discussing in detail how this occurs.

The DAMA experiment [1] observes an annual modulation in their count rate, which can be parametrized as

$$
R_{i}=R_{i}^{0}+S_{i}^{1} \cos \left[\omega\left(t-t_{0}\right)\right] .
$$

The subscript $i$ in this expression denotes different energy bins. The constant term $R_{i}^{0}$ is composed of both a signal component coming from dark matter initiated processes, and a background component arising from other sources of nuclear recoil: $R_{i}^{0}=b_{i}^{0}+S_{i}^{0}$. The expressions for $S_{i}^{0}$ and $S_{i}^{1}$ are obtained by integrating Eq. (4) over a given energy bin.

Channeling is a potentially important but difficult-topredict theoretical effect which can significantly change the interpretation of DAMA's signal, especially when comparing this signal to the results of other direct detection experiments. In a typical nuclear recoil event, only a fraction of the total energy is detected (as a combination of scintillation light, heat, and ionization, depending on the detector). The ratio of the observed energy to the total recoil energy is known as the quenching factor. For crystal scintillators, however, such as those used by the DAMA Collaboration, a portion of the events will be "channeled," causing most of the recoil energy in those events to be observed (effectively changing the quenching factor to $q \approx$ $1)$. This occurs when the incident particle interacts only electromagnetically with the scintillator material, which can occur for certain energies and incidence angles. The importance of this effect for the DAMA experiment was first discussed in Ref. [21], and an analysis of its effect was performed by the DAMA Collaboration in Ref. [28]; we refer the reader to these references for a more detailed discussion.

Questions of how to quantitatively account for the effects of channeling in DAMA have made efforts to interpret their results, and to compare their results to those of other experiments, somewhat difficult. In particular, the 
compatibility of the DAMA modulation with the CoGeNT and CDMS excesses (and the compatibility of DAMA with the null results of XENON and other searches) depends strongly on the fraction of elastic scattering events which are channeled rather than quenched. An estimate of the fraction of channeled events based on simulations is given in Fig. 4 of Ref. [28]. We use the following simple parametrization in our analysis [29]:

$$
f_{N a} \approx \frac{1}{1+1.14 E_{R}(\mathrm{keV})}, \quad f_{I} \approx \frac{1}{1+0.75 E_{R}(\mathrm{keV})} .
$$

It should be noted, however, that channeling at DAMA has not been experimentally verified, and studies of $\mathrm{NaI}$ at energies above the region of interest for DAMA have failed to find such an effect [30]. We also consider the DAMA preferred region if channeling is not present. In this case, scattering off of iodine cannot reproduce the DAMA signal at the low masses of interest. Scattering off of sodium is relevant, however, and is shown as a yellow, lightly shaded region in each frame of Fig. 2. The cross sections required to explain DAMA without any channeling are somewhat too high to explain CoGeNT, but the range of masses needed to explain the two experiments is surprisingly similar.

In Fig. 2, we show alongside the CoGeNT region the parameter space that can accommodate DAMA's annual modulation signal. In addition to the DAMA and CoGeNT regions, we also show the region of the parameter space in which the 2 events observed by CDMS can be generated (consistent with the small excess recently reported [2]). If the actual fraction of channeled events is smaller than its estimated value in Eq. (6), then the DAMA region simply shifts upward in cross section to accommodate the change. Thus, in the left panel of Fig. 1, one can see by eye how modifying the channeled fraction to $60 \%$ of its estimated value easily moves the DAMA region into better agreement with CoGeNT. In the right panel of Fig. 1 we also see how $v_{0}$ shifts the DAMA region to lower masses. The two constraint plots in Fig. 1 are considerably more favorable for a light elastically scattering WIMP interpretation of DAMA than previous results [31], the reason for this being that the allowed region of parameter space depends quite sensitively on assumptions about the halo model and the XENON10 scintillation efficiency. We next turn our attention to these details and how they impact the constraints from CDMS and XENON10.

\section{B. Consistency with null results}

In the light WIMP window, the null results of XENON10 are among the most constraining, due to its relatively low threshold of $4.5 \mathrm{keV}$. Recently that threshold was lowered further to $2 \mathrm{keV}$ [11], although no constraint was derived on low mass, elastically scattering dark matter in that analysis. To derive the XENON10 constraint, software efficiencies given in Ref. [11] are coupled with an $S_{2}$
12 electron efficiency and an $S_{1}$ detector acceptance efficiency taken from Ref. [32]. At face value, the XENON10 constraint appears to completely rule out the regions of the low mass window favored by DAMA and CoGeNT.

There are reasons to be skeptical of such a conclusion, however. In particular, a new measurement was recently made of the scintillation efficiency (the fraction of nuclear recoil energy that goes into scintillation light) of liquid xenon, $L_{\text {eff }}$ [33]. According to the new measurement, the scintillation efficiency is significantly lower than previously reported by the XENON10 Collaboration, who take $L_{\text {eff }}=0.19$ in their analysis. A lower scintillation efficiency translates to a reduced sensitivity of xenon-based detectors to light dark matter. Although the central values of the new $L_{\text {eff }}$ measurement still lead to considerable tension with the region implied by CoGeNT, if we adopt values of $L_{\text {eff }}$ which are $1 \sigma$ below its central values, we find that consistent regions can be found. This constraint is included in Fig. 1, along with the constraint from the CDMS silicon analysis [15,16]. We show in Fig. 1, for comparison, the constraints from XENON10 using the old $L_{\text {eff }}=0.19$, alongside those using the new measurement with and without taking into account the $1 \sigma$ uncertainty in each bin. Note that the XENON10 excluded region changes rather drastically with $L_{\text {eff }}$. Although this allows us to conclude that the CoGeNT region is consistent with the XENON10 constraint, the region favored by DAMA still appears to be disfavored by XENON10. The most important measurements of $L_{\text {eff }}$ are in the energy bins near XENON10's threshold, since these determine the lowest recoil energies that XENON10 can probe. For illustration, we also show in Fig. 1 the constraint taking the $-\frac{1}{2} L_{\text {eff }}$ values only in the three bins centered on 4.9, 5.7, and $6.4 \mathrm{keV}$ (and $-\frac{1}{4} L_{\text {eff }}$ at $3.9 \mathrm{keV}$ so that scintillation always increases with recoil energy), and central values elsewhere.

The second significant effect that we have taken into account is shifts from uncertainty in the halo model. It is generally considered that $v_{0}=220 \mathrm{~km} / \mathrm{s}$ is a useful starting point for the halo model; however, if the distribution of velocities is broader, then lighter dark matter will be favored, bringing the DAMA region below the XENON10 constraint curve. Taking $v_{0}=270 \mathrm{~km} / \mathrm{s}$, we see in the right frame of Fig. 1 that the region consistent with DAMA shifts to lower masses, and becomes consistent with both the constraints of the null experiments and with the CoGeNT preferred region.

To further investigate the source of the remaining tension, we examine the effects of both removing the lowest bin from the DAMA data sample, and making the errors in those bins a factor of 2 larger. In the left frame of Fig. 3, we have modified the DAMA constraint by showing the region when the lowest bin is neglected altogether (grey, $90 \%$ and 99\% constraints), as well as the region when the errors are doubled on the lowest two bins (again, for $90 \%$ and $99 \%$ 

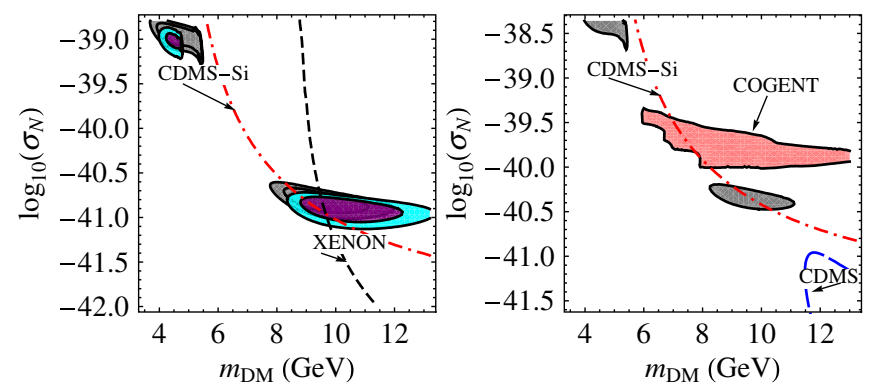

FIG. 3 (color online). Left panel: The DAMA region and XENON10 constraint are the same as in the right panel of Fig. 1 but, for illustration, with the effects of removing the lowest bin of data from the DAMA region (gray region), or with the effects of increasing the errors in those bins by a factor of 2 (90\% and $99 \%$ confidence levels). We see that the DAMA region is shifted to smaller masses and becomes more consistent with the XENON10 constraint. Right panel: The same as in the left frame, but with dark matter couplings only to neutrons, $f_{n}=$ 1 and $f_{p}=0$. The constraints from CDMS silicon relative to the DAMA region are weaker than for $f_{n}=f_{p}$. Only the $90 \%$ confidence limit for DAMA removing the lowest bin is shown.

TABLE I. Relevant features of the null experiments used in our analysis. Efficiencies and cut acceptances are taken from the references above.

\begin{tabular}{lcccc}
\hline \hline Experiment & Target & Exposure $(\mathrm{kgd})$ & Threshold & Ref. \\
\hline CDMS-II & $\mathrm{Ge}$ & 612.0 & $10 \mathrm{keV}$ & {$[66]$} \\
& $\mathrm{Si}$ & 83.3 & $7 \mathrm{keV}$ & {$[15,16]$} \\
XENON10 & $\mathrm{Xe}$ & 316.0 & $2 \mathrm{keV}$ & {$[11]$} \\
& & with $L_{\text {eff }}=0.19$ & \\
\hline \hline
\end{tabular}

confidence levels). We can see that most of the remaining tension is removed: much of the inconsistency seems to stem from the lowest one to two bins in the DAMA data.

In the results shown so far, we have assumed that the couplings of the dark matter to protons are equal to those to neutrons. If the dark matter couples to neutrons more strongly than to protons, we can further relax the constraints of the CDMS silicon analysis. For example, taking $f_{n}=1, f_{p}=0$ we find the results shown in the right frame of Fig. 3 (see Table I for the parameters used for null results).

\section{MODELS OF LOW MASS DARK MATTER}

We begin this section by carrying out a relatively model independent operator analysis of the low mass window relevant for CoGeNT, DAMA, and CDMS. We then turn to discussing two classes of models: those arising from models of ADM, and from supersymmetry. ${ }^{3}$

\footnotetext{
${ }^{3}$ Singlet scalars [34], mirror models [35], and "WIMPless" models [36] also give rise to light WIMPs, though we do not discuss those models here. We refer the reader to these references for details.
}

\section{A. Operator analysis}

The operators connecting dark sector Dirac fermions $\chi$ or complex scalars $\phi$ to visible sector fermions $f$ are

$$
\begin{gathered}
\mathcal{L}_{f S}=\frac{G_{f}}{\sqrt{2}} \bar{\chi} \chi \bar{f} f, \\
\mathcal{L}_{f V}=\frac{G_{f}}{\sqrt{2}} \bar{\chi} \gamma^{\mu} \chi \bar{f} \gamma_{\mu} f, \\
\mathcal{L}_{s S}=\frac{F_{f}}{\sqrt{2}} \phi^{*} \phi \bar{f} f, \\
\mathcal{L}_{s V}=\frac{F_{f}}{\sqrt{2}} \phi^{*} \stackrel{\leftrightarrow}{\partial}_{\mu} \phi \bar{f} \gamma^{\mu} f .
\end{gathered}
$$

\section{Fermionic dark matter}

For fermionic dark matter, the scattering cross section obtained from these operators is given by

$$
\sigma=\frac{4 a}{\pi} \frac{m_{\mathrm{DM}}^{2} m_{N}^{2}}{\left(m_{\mathrm{DM}}+m_{N}\right)^{2}}\left(Z f_{p}+(A-Z) f_{n}\right)^{2}
$$

where $A$ and $Z$ are the atomic mass and atomic number of the target nuclei, and $a$ is a number dependent on whether the fermion is Dirac of Majorana. For Majorana fermions $a=1$, while for Dirac fermions, $a=1 / 4$. The effective couplings to protons and neutrons, $f_{p, n}$, can be written in terms of the WIMP's couplings to quarks and depend both on the spin of the DM particle and the mediator. In terms of the dark matter's effective couplings, we can write

$$
f_{p, n}=\sum_{q=u, d, s} \frac{G_{q}}{\sqrt{2}} f_{T q}^{(p, n)} \frac{m_{p, n}}{m_{q}}+\frac{2}{27} f_{T G}^{(p, n)} \sum_{q=c, b, t} \frac{G_{q}}{\sqrt{2}} \frac{m_{p, n}}{m_{q}},
$$

where $G_{q}$ denotes the dark matter's effective coupling to a given quark species. The first term reflects scattering with light quarks, while the second term accounts for interactions with gluons through a heavy-quark loop. The values of $f_{T_{q}}^{(p, n)}$ are proportional to the matrix element, $\langle\bar{q} q\rangle$, of quarks in a nucleon. In our numerical calculations, we use values for these quantities based on recent lattice QCD results [37,38]. If the dark matter scatters with nuclei through a scalar interaction, both of the terms in Eq. (12) typically yield sizable contributions to the cross section. If, on the other hand, the dark matter scatters through a vector interaction, then heavy-quark loop contribution is negligible, and the nucleon level couplings simplify to

$$
f_{p}=2 \frac{G_{u}}{\sqrt{2}}+\frac{G_{d}}{\sqrt{2}}, \quad f_{n}=\frac{G_{u}}{\sqrt{2}}+2 \frac{G_{d}}{\sqrt{2}} .
$$

The effective operator relates to the parameters in the underlying Lagrangian through 


$$
\frac{G_{f}}{\sqrt{2}}=\frac{g_{\mathrm{DM}} g_{f}}{M_{\psi}^{2}},
$$

where $\psi$ denotes the mediator, and $g_{\mathrm{DM}}, g_{f}$ denote the mediator's couplings to the dark matter and standard model fermion, respectively.

The annihilation cross sections for Dirac fermions $\bar{\chi} \chi \rightarrow \bar{f} f$, through scalar and vector mediators, are

$$
\begin{gathered}
\sigma_{\mathrm{ann}}^{f S} v=\frac{v^{2} m_{\mathrm{DM}}^{2}}{16 \pi} \sum_{f} G_{f}^{2} c_{f}\left(1-m_{f}^{2} / m_{\mathrm{DM}}^{2}\right)^{3 / 2}, \\
\sigma_{\mathrm{ann}}^{f V} v=\frac{m_{\mathrm{DM}}^{2}}{4 \pi} \sum_{f} G_{f}^{2} c_{f}\left(1-m_{f}^{2} / m_{\mathrm{DM}}^{2}\right)^{1 / 2} \\
\times\left[\left(2+\frac{m_{f}^{2}}{m_{\mathrm{DM}}^{2}}\right)+\left(\frac{8-4 \frac{m_{f}^{2}}{m_{\mathrm{DM}}^{2}}+5 \frac{m_{f}^{4}}{m_{\mathrm{DM}}^{4}}}{24\left(1-\frac{m_{f}^{2}}{m_{\mathrm{DM}}^{2}}\right)}\right) v^{2}\right] .
\end{gathered}
$$

\section{Scalar dark matter}

There are related expressions for scalar dark matter scattering and annihilation cross sections:

$$
\sigma=\frac{a}{\pi} \frac{m_{N}^{2}}{\left(m_{\mathrm{DM}}+m_{N}\right)^{2}}\left(Z f_{p}+(A-Z) f_{n}\right)^{2},
$$

with

$$
f_{p, n}=\sum_{q=u, d, s} \frac{F_{q}}{\sqrt{2}} f_{T q}^{(p, n)} \frac{m_{p, n}}{m_{q}}+\frac{2}{27} f_{T G}^{(p, n)} \sum_{q=c, b, t} \frac{F_{q}}{\sqrt{2}} \frac{m_{p, n}}{m_{q}},
$$

for scalar mediators, and

$$
f_{p}=2 \frac{F_{u}}{\sqrt{2}}+\frac{F_{d}}{\sqrt{2}}, \quad f_{n}=\frac{F_{u}}{\sqrt{2}}+2 \frac{F_{d}}{\sqrt{2}},
$$

for vector mediators. For scalar mediators, $a=1(1 / 4)$ for real (complex) scalars, and for vector mediators, $a=4(1)$ for real (complex) scalars.

For scalar mediators, the effective coupling is related to the underlying parameters in the Lagrangian through

$$
\frac{F_{f}}{\sqrt{2}}=\frac{g_{\mathrm{DM}} y_{f}\langle H\rangle}{M_{h}^{2}},
$$

where $v$ is the Higgs vacuum expectation value (VEV), $v / \sqrt{2}=\langle H\rangle=174 \mathrm{GeV}$, and $M_{h}$ is its mass, derived from a term in the Lagrangian

$$
\mathcal{L}=g_{\mathrm{DM}} H^{\dagger} H S^{\dagger} S+y_{f} H \bar{f} f .
$$

For vector mediators (and complex scalars), the effective coupling is related to the underlying parameters through

$$
\frac{F_{f}}{\sqrt{2}}=\frac{g_{\mathrm{DM}} g_{f} m_{\mathrm{DM}}}{M_{\psi}^{2}} .
$$

The corresponding annihilation cross sections for a complex scalar $\bar{\phi} \phi \rightarrow \bar{f} f$, through real scalar and vector mediators, are

$$
\begin{gathered}
\sigma_{\mathrm{ann}}^{s S} v=\frac{1}{8 \pi} \sum_{f} F_{f}^{2} c_{f}\left(1-m_{f}^{2} / m_{\mathrm{DM}}^{2}\right)^{1 / 2} \\
\times\left[\left(1-\frac{m_{f}^{2}}{m_{\mathrm{DM}}^{2}}\right)+\left(\frac{3 m_{f}^{2}}{8 m_{\mathrm{DM}}^{2}}\right) v^{2}\right], \\
\sigma_{\mathrm{ann}}^{S V} v=\frac{v^{2}}{12 \pi} \sum_{f} F_{f}^{2} c_{f}\left(1-m_{f}^{2} / m_{\mathrm{DM}}^{2}\right)^{1 / 2}\left(1+\frac{m_{f}^{2}}{2 m_{\mathrm{DM}}^{2}}\right) .
\end{gathered}
$$

\section{CoGeNT and thermal WIMPs}

Beginning with the case of a fermionic WIMP, we can consider either scalar or vector interactions with quarks to produce a spin-independent elastic scattering with nuclei. Following Ref. [39], we show results for each of these cases in Fig. 4. In the upper left frame, we have assumed that the dark matter's effective scalar couplings to fermions are proportional to the mass of the fermion, $G_{f} \propto m_{f}$, but are otherwise universal. To produce an elastic scattering cross section capable of generating the excesses observed by CoGeNT and DAMA (shown as a dotted region), we need an effective coupling of $G_{\text {eff }} \times\left(1 \mathrm{GeV} / m_{f}\right) \sim$ $2 \times 10^{-6} \mathrm{GeV}^{-2}$. If this is the only interaction experienced by the WIMP, however, it will be significantly overproduced in the early universe, resulting in a thermal relic abundance in excess of the measured abundance of dark matter (see e.g. [34,40] for related conclusions concerning the DAMA favored cross sections). The solid lines in each frame of Fig. 4 denote the effective coupling strength that leads to the desired thermal dark matter abundance, assuming no other interactions further deplete the relic density. For details pertaining to the dark matter's annihilation cross section and the thermal relic abundance calculation, see Ref. [39]. Note that we use a temperature dependent number of relativistic degrees of freedom in our relic abundance calculation (see, for example, Ref. [41]).

This apparent conflict between the couplings required to produce the desired elastic scattering rate and the measured dark matter abundance can easily be resolved, however, if the fermionic WIMP also annihilates through other process, such as through pseudoscalar or axial interactions (which do not, in turn, generate a contribution to the spinindependent elastic scattering cross section). In the upper right frame of Fig. 4, we show the effective pseudoscalar coupling that leads to the desired dark matter abundance. A $9 \mathrm{GeV}$ WIMP, for example, with effective scalar and pseudoscalar couplings of $G_{\text {eff }} \sim 3 \times 10^{-6} \mathrm{GeV}^{-2} \times$ $\left(m_{f} / 1 \mathrm{GeV}\right)$ would satisfy both the elastic scattering and relic density requirements. Alternatively, one could also consider effective couplings that are not universal across 

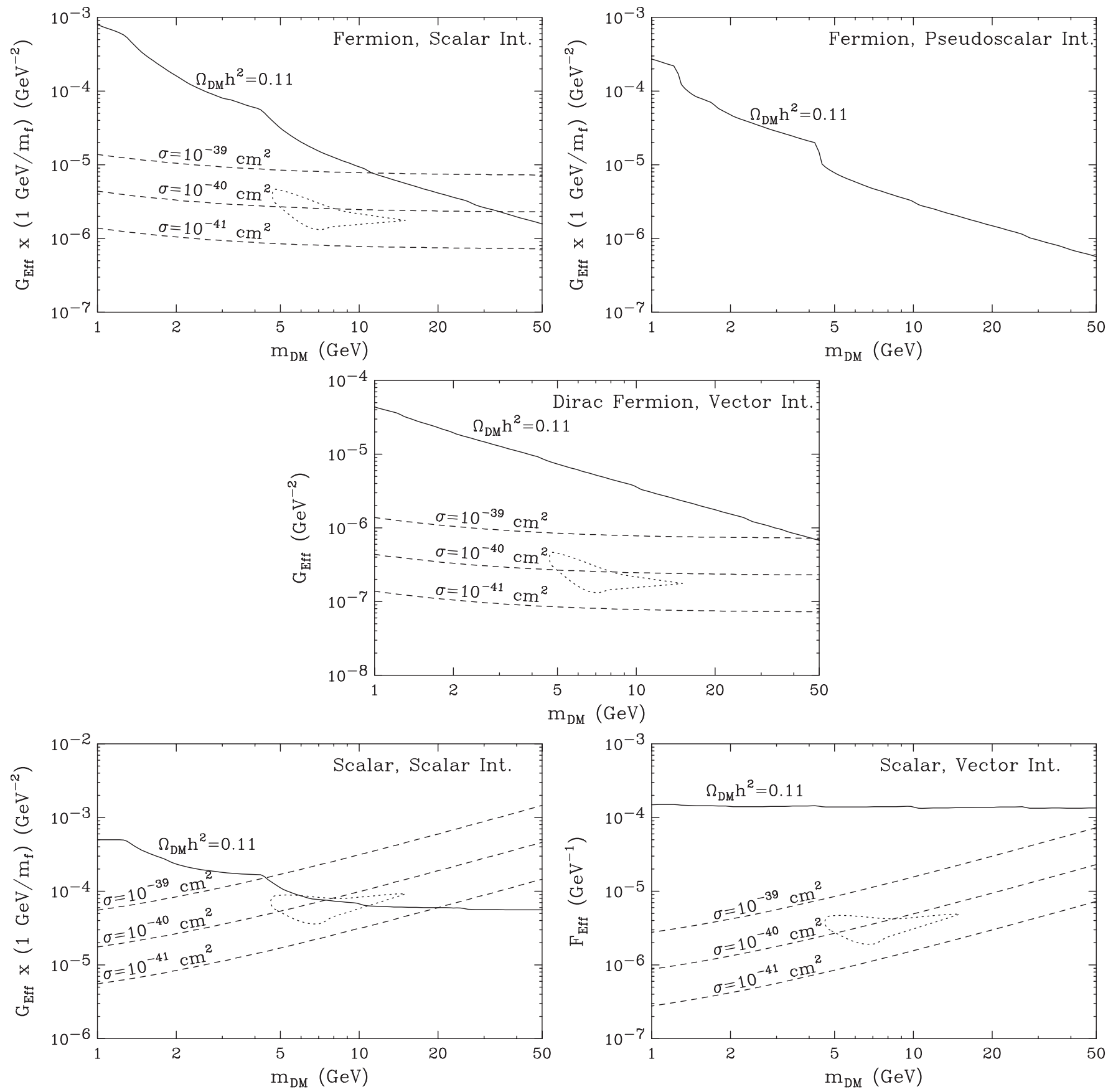

FIG. 4. The effective dark matter-fermion couplings required to generate a given spin-independent elastic scattering cross section with nucleons (dashed lines), and to produce a thermal abundance consistent with the observed dark matter density (solid lines). In each frame, the dotted region represents the approximate range of couplings and masses that leads to a signal consistent with that reported by CoGeNT. The various frames show results for a fermionic dark matter particle with scalar interactions (upper left panel), pseudoscalar interactions (upper right panel), vector interactions (center panel), or a scalar dark matter particle with scalar interactions (lower left panel), or vector interactions (lower right panel). In the case of a scalar dark matter particle with approximately universal scalar couplings to standard model fermions, the coupling size required to generate the excesses observed by CoGeNT and DAMA is also approximately the size required to thermally generate the observed density of dark matter in the early universe.

all fermion species. If the dark matter couples preferentially to leptons, for example, its relic abundance could be reduced without increasing the elastic scattering cross section.
As a simple example, we could consider a light fermionic dark matter particle that couples to quarks through the exchange of the standard model Higgs boson. This scenario, however, is unlikely to be able to generate the 
excesses reported by CoGeNT and DAMA. In particular, even if we take the dark matter's couplings to the Higgs to be of order unity, the effective couplings to quarks would only be $G_{\text {eff }} \sim 4 \times 10^{-7} \mathrm{GeV}^{-2}\left(m_{f} / 1 \mathrm{GeV}\right)$, or approximately an order of magnitude smaller than is needed to generate the observed number of events. From this example, we see that the mediator must either be considerably lighter than the standard model Higgs collider limits, or must have coupling to quarks that are larger than those of the standard model Higgs.

If the dark matter is a Dirac fermion, vector couplings can also generate a spin-independent elastic scattering cross section. In the center frame of Fig. 4, we show the effective couplings required in this case. Here, we have assumed that $G_{\text {eff }}$ is the same for all species of standard model fermions. Once again, we find that the couplings required to generate the desired elastic scattering rate are not sufficient to yield the observed dark matter abundance, and other interactions are required to avoid the overproduction of dark matter in the early universe.

If instead of a fermion, the WIMP is a scalar particle, we again can rely on either scalar or vector interactions as the source of the CoGeNT and DAMA excesses. In the case of scalar interactions, we find that the effective coupling required to generate the elastic scattering cross section implied by CoGeNT and DAMA also leads to a thermal relic abundance consistent with the observed dark matter density (see the lower left frame of Fig. 4). So, in this simplest case we have considered (a scalar dark matter particle with approximately universal scalar couplings to standard model fermions), we find that if we set the couplings to the size required to generate the observed density of dark matter, then we also predict an elastic scattering rate that can easily account for the CoGeNT and DAMA excesses (see [34] for earlier work interpreting this part of parameter space in the context of DAMA). A scalar WIMP with vector interactions, on the other hand, requires another interaction to avoid being overproduced in the early universe (see the lower right frame of Fig. 4).

\section{B. Asymmetric dark matter}

In most dark matter models, the abundance of dark matter and the baryon asymmetry of the universe are unrelated to each other. The dark matter density is generally determined by its mass and self-annihilation cross section, which determine the temperature at which it thermally freezes out in the early universe. The baryon asymmetry, in contrast, is set by $C P$ violating phases, and by the strength of the electroweak phase transition. Within this paradigm, the densities of dark matter and of baryons in the universe have nothing to do with one another. But despite this expectation, observations have revealed that the cosmological densities of these two sectors are similar:

$$
\frac{\rho_{\mathrm{DM}}}{\rho_{b}} \approx 5 \text {. }
$$

Within the context of most thermal dark matter models, one must view this similarity as a coincidence (unless anthropic arguments are invoked).

Motivated by this apparent coincidence, models in which the dark matter abundance is tied to the baryon asymmetry have been developed [12,13]. In ADM models, a mechanism (in some cases a higher dimension operator) enforces a relationship between the dark matter and baryon chemical potentials:

$$
c_{1}\left(n_{X}-n_{\bar{X}}\right)=n_{b}-n_{\bar{b}},
$$

where $c_{1}$ is a number $\mathcal{O}(1)$ whose precise value depends on the operator transferring the asymmetry.

Once the symmetric thermal abundance, $n_{X}+n_{\bar{X}}$, has annihilated away, we are left with a relationship between the asymmetric relic dark matter density and the relic baryon number density, implying

$$
m_{\mathrm{DM}}=5 c_{1} m_{p},
$$

which combined with Eq. (25) requires that the dark matter's mass is roughly within the range of $\sim 1-10 \mathrm{GeV}$.

In Ref. [13], a class of models was discussed in detail that fits naturally within the CoGeNT, DAMA, and CDMS windows. In one such model (a supersymmetric example), the dark matter and baryon chemical potentials are related to each other through a superpotential interaction

$$
W=\frac{\bar{X}^{2} L H}{M} .
$$

This operator transfers a lepton asymmetry to the dark matter and predicts a dark matter mass of approximately $m_{X} \sim 11 \mathrm{GeV}$. Other classes of models have also been discussed in which the dark matter is related to the baryon density through the out-of-equilibrium decay of a new heavy particle to both sectors.

ADM thus predicts dark matter with a mass near or within the window implied by CoGeNT and DAMA. In order to provide an explanation for these excesses, however, such a dark matter candidate must also possess an appropriately large elastic scattering cross section. The magnitude of this cross section is determined not only by the properties of the dark matter particle itself, however, but also on the mass and couplings of the mediator, which makes it a somewhat more model dependent quantity. As one possibility (as discussed in Ref. [13]), a simple $Z^{\prime}$ mediator with mass around the $\mathrm{TeV}$ scale gives rise to a scattering cross section of the magnitude observed by CoGeNT and DAMA. Alternatively, a singlet scalar that mixes with the Higgs may also give rise to an appropriately sized elastic scattering cross section. This singlet may be responsible for the mass of the singlet dark matter through an operator

$$
W_{M}=\lambda S \bar{X} X,
$$

and a mixing with the Higgs through the usual NMSSM 
term

$$
W_{n}=\zeta S H_{u} H_{d}
$$

gives rise to the appropriate size scattering cross section for CoGeNT and DAMA [10]:

$$
\begin{aligned}
\sigma_{N} \approx & \frac{\mu_{n}^{2}}{\pi} N_{n}^{2}\left(\frac{\lambda \zeta v_{u}\langle S\rangle}{m_{h^{0}}^{2}}\right)^{2} \frac{1}{m_{S}^{4}} \\
\approx & 3 \times 10^{-41} \mathrm{~cm}^{2}\left(\frac{N_{n}}{0.1}\right)^{2}\left(\frac{\lambda}{0.2}\right)^{2}\left(\frac{\zeta}{10^{-2}}\right)^{2}\left(\frac{\langle S\rangle}{20 \mathrm{GeV}}\right)^{2} \\
& \times\left(\frac{100 \mathrm{GeV}}{m_{h^{0}}}\right)^{4}\left(\frac{10 \mathrm{GeV}}{m_{S}}\right)^{4},
\end{aligned}
$$

where $N_{n}$ arises from the effective coupling of the Higgs to the target nucleus. ${ }^{4}$

\section{C. (Nonminimal) neutralino dark matter}

Within the context of the minimal supersymmetric standard model (MSSM), light neutralino dark matter can coherently scatter with nuclei through scalar Higgs exchange (and to a lesser extent, through squark exchange), while annihilating fairly efficiently through pseudoscalar Higgs exchange (which, unlike scalar exchange, is not $s$-wave suppressed). The composition and couplings of a neutralino lighter than $m_{Z} / 2$ are constrained by the LEP measurements of the invisible $Z$ branching fraction, which require $\Gamma_{Z \rightarrow \chi^{0} \chi^{0}}<3 \mathrm{MeV}$ (at $2 \sigma$ C.L.) [42]. This approximately translates to a bound of $\left|N_{13}^{2}-N_{14}^{2}\right| \lesssim 0.13$ at $2 \sigma$, where $N_{13}^{2}$ and $N_{14}^{2}$ are the up-type and down-type Higgsino fractions of the lightest neutralino. If we maximize the neutralino-neutralino-Higgs couplings by saturating this bound, and impose the constraints from the Tevatron for Higgs boson production at large $\tan \beta$, it has been shown that the largest scattering cross section that can be achieved is $\sim 2 \times 10^{-41} \mathrm{~cm}^{2}$ [43]. This is near to the CoGeNT and DAMA regions $[40,44]$, but certainly on the lower edge.

To obtain larger effective couplings (and thus larger elastic scattering and annihilation cross sections), one could consider interactions mediated by Higgs bosons which are lighter than those allowed within the MSSM. Within the context of the NMSSM, for example, light ( 1-100 GeV) scalar and pseudoscalar Higgs bosons can exist, while not conflicting with existing collider constraints, as a result of their reduced couplings to standard model fields. In particular, if the lightest scalar and pseudoscalar Higgs bosons contain a significant admixture of the Higgs singlet, such particles can be much lighter than the MSSM Higgs limits, increasing the corresponding effective couplings of the lightest neutralino $[45,46]$.

\footnotetext{
${ }^{4}$ Note that the relic abundance arguments described in Sec. III A do not apply in the case of asymmetric dark matter. Furthermore, the indirect detection prospects described in Sec. IV do not apply to this class of dark matter candidates.
}

As an example, we could consider a 5-10 GeV neutralino which is mostly bino, but with a $\sim 5 \%$ Higgsino admixture. If such a particle annihilates through the exchange of $\mathrm{a} \lesssim 70 \mathrm{GeV}$ mixed singlet-MSSM-like pseudoscalar Higgs, and if $\tan \beta$ is relatively large $(\sim 30-50)$, then we find that this process can yield the desired relic abundance. Furthermore, if a down-type scalar Higgs $\left(H_{1}\right)$ were also of a comparable mass, and comparably mixed with the Higgs singlet, it would mediate an elastic scattering cross section consistent with the rate observed by CoGeNT and DAMA. This example serves as an illustration of the more general conclusion that the range of masses and scattering cross sections implied by CoGeNT and DAMA requires a particle or particles that are either relatively light or possess relatively large couplings to mediate the interactions of the dark matter.

\section{IMPLICATIONS FOR INDIRECT DETECTION}

Regions with high densities of dark matter are in many cases predicted to produce potentially observable fluxes of dark matter annihilation products. As the annihilation rate of dark matter scales with the square of the inverse mass of the particle, the prospects for indirect detection are particularly encouraging for dark matter masses as light as we are considering in this paper. In this section, we discuss some of the implications of the CoGeNT and DAMA excesses for indirect dark matter searches using neutrinos, gamma rays, and charged cosmic rays. We also comment on the possibility that white dwarf stars could be used as a probe of dark matter in models capable of generating the observed excesses.

\section{A. Neutrinos from dark matter annihilations in the Sun}

If dark matter particles are present in the vicinity of the solar system, they will scatter elastically with and become captured in the Sun at a rate approximately given by [47]

$$
\begin{aligned}
C^{\odot} \approx & 1.3 \times 10^{24} \mathrm{~s}^{-1}\left(\frac{\rho_{0}}{0.3 \mathrm{GeV} / \mathrm{cm}^{3}}\right)\left(\frac{270 \mathrm{~km} / \mathrm{s}}{\bar{v}}\right) \\
& \times\left(\frac{10 \mathrm{GeV}}{m_{\mathrm{DM}}}\right) \sum_{A} F_{A}\left(\frac{\sigma_{A}}{A^{2} \times 10^{-40} \mathrm{~cm}^{2}}\right) S\left(m_{\mathrm{DM}} / m_{A}\right),
\end{aligned}
$$

where $\rho_{0}$ is the local dark matter density, $\bar{v}$ is the local root-mean-square velocity of halo dark matter particles, and $m_{\mathrm{DM}}$ is the dark matter mass. The sum is over the mass number of the chemical species present in the Sun, and the quantity $F_{A}$ contains information pertaining to the solar abundances, dynamical factors, and form factor suppression of each element $\left(F_{\mathrm{H}}=1, F_{\mathrm{He}} \approx 1.1\right.$, etc.). The quantity $S$ is a kinetic suppression factor given by

$$
S(x)=\left[\frac{A(x)^{3 / 2}}{\left(1+A(x)^{3 / 2}\right)}\right]^{2 / 3},
$$


where

$$
A(x)=\frac{3 x}{2(x-1)^{2}}\left(\frac{\left\langle v_{\mathrm{esc}}\right\rangle}{\bar{v}}\right)^{2} .
$$

The evolution of the number of dark matter particles in the Sun, $N$, is described by

$$
\dot{N}=C^{\odot}-A^{\odot} N^{2}-E^{\odot} N,
$$

where $C^{\odot}$ is the capture rate, $A^{\odot}$ is the annihilation cross section times the relative WIMP velocity per unit volume, and $E^{\odot}$ is the inverse time for a WIMP to exit the Sun via evaporation (which is negligible for dark matter particles heavier than approximately $3-4 \mathrm{GeV}[48,49]) . A^{\odot}$ can be approximated by

$$
A^{\odot}=\frac{\langle\sigma v\rangle}{V_{\mathrm{eff}}},
$$

where $V_{\text {eff }}$ is the effective volume of the core of the Sun determined roughly by matching the core temperature with the gravitational potential energy of a single WIMP at the core radius. This was found in Refs. [49,50] to be

$$
V_{\mathrm{eff}}=5.7 \times 10^{30} \mathrm{~cm}^{3}\left(\frac{1 \mathrm{GeV}}{m_{\mathrm{DM}}}\right)^{3 / 2} .
$$

Neglecting evaporation, the present WIMP annihilation rate in the Sun is given by

$$
\Gamma=\frac{1}{2} A^{\odot} N^{2}=\frac{1}{2} C^{\odot} \tanh ^{2}\left(t_{\odot} \sqrt{C^{\odot} A^{\odot}}\right),
$$

where $t_{\odot} \approx 4.5 \times 10^{9} \mathrm{yr}$ is the age of the Solar System. For a 5-10 GeV WIMP, capture-annihilation equilibrium is reached so long as the WIMP's annihilation cross section is larger than $\sigma v \geqslant 10^{-30} \mathrm{~cm}^{3} / \mathrm{s}$. Once equilibrium is reached, the final annihilation rate (and corresponding neutrino flux and event rate) has no further dependence on the dark matter particle's annihilation cross section.

As the dark matter particles annihilate, they can generate neutrinos through a wide range of channels. Annihilations to bottom quarks, charm quarks, and tau leptons each generate neutrinos in their subsequent decays. In some models, dark matter particles can also annihilate directly to neutrino pairs. Once produced, neutrinos travel to the Earth where they can be detected. For the mass range of interest here, the strongest constraints on the capture and annihilation rate in the Sun come from the SuperKamiokande experiment [51]. ${ }^{5}$ In Fig. 5, we show the constraints on a light dark matter particle from SuperKamiokande (adapted from Ref. [53]). From this figure, we conclude that if a dark matter particle responsible for the excess observed by CoGeNT annihilates primarily to neutrinos or tau leptons, it should have produced a flux of neutrinos observable to Super-Kamiokande. If the dark

\footnotetext{
${ }^{5}$ See also [52], which discusses Super-Kamiokande constraints on WIMPless dark matter DAMA interpretations.
}

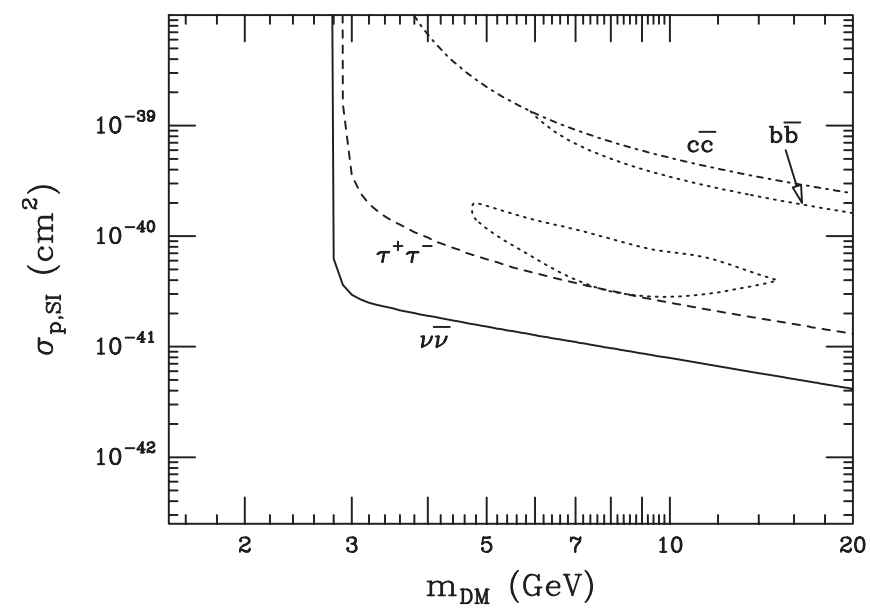

FIG. 5. The upper limit on a light WIMP's spin-independent elastic scattering cross section with nuclei from the SuperKamiokande experiment, for various choices of the dominant annihilation channel. Also shown is the region of the plane which provides a good fit to the excess observed by CoGeNT. From this figure, we conclude that Super-Kamiokande excludes a dark matter particle which annihilates entirely to neutrinos or taus as a source of the CoGeNT excess. This figure was adapted from Ref. [53].

matter annihilates significantly to the other, it could produce the rates observed at CoGeNT and DAMA without coming into conflict with Super-Kamiokande's observations. Alternatively, if the dark matter's annihilation cross section is highly suppressed (such as in the case of asymmetric dark matter, as discussed in Sec. III B), then these constraints do not apply.

Note that the results shown in Fig. 5 differ somewhat from those found in Ref. [53]. This is because, in Ref. [53], only scattering off of hydrogen and helium nuclei was included, and a simplified treatment of the velocity distribution was adopted [54]. The capture rates used to obtain the limits found in Fig. 5 are in good agreement with the publicly available code DARKSUSY [55].

\section{B. Gamma ray searches from dark matter}

In generality, the flux of gamma rays from dark matter annihilations can be written as

$$
\Phi_{\gamma}\left(E_{\gamma}, \psi\right)=\frac{1}{2} \sigma|v| \frac{d N_{\gamma}}{d E_{\gamma}} \frac{1}{4 \pi m_{\mathrm{DM}}^{2}} \int_{\mathrm{los}} \rho^{2} d l,
$$

where $\sigma|v|$ is the dark matter's, $\psi$ is the angle observed, $\rho$ is the dark matter density, and the integral is performed over the line of sight being observed. $d N_{\gamma} / d E_{\gamma}$ is the gamma ray spectrum generated per WIMP annihilation, which depends on the mass of the dark matter particle and on its dominant annihilation channels. The integral in Eq. (39) depends on the distribution of dark matter and is largest in the directions of the sky where very dense and relatively nearby concentrations of dark matter are present. 
The brightest source of gamma rays from dark matter annihilation is generally expected to be the region around the center of the Milky Way [56]. Nearby dwarf spheroidal galaxies are also very promising sources for indirect detection [57].

To estimate the gamma ray flux from the Galactic center region, we adopt a dark matter distribution which follows the commonly used Navarro-Frenk-White (NFW) profile [58]:

$$
\rho(r)=\frac{\rho_{0}}{(r / R)[1+(r / R)]^{2}},
$$

where $r$ is the distance from the Galactic center, $R \sim$ $20 \mathrm{kpc}$ is the scale radius, and $\rho_{0}$ is fixed by imposing that the dark matter density at the distance of the Sun from the Galactic center is equal to the value approximately inferred by observations $\left(\sim 0.3 \mathrm{GeV} / \mathrm{cm}^{3}\right)$.

In Fig. 6, we show the spectrum of gamma rays from dark matter annihilations in the inner Milky Way, and compare this to the spectrum measured by the FGST [8]. The curves shown in each frame are for the case of a $9 \mathrm{GeV}$ dark matter particle annihilating with a cross section of $\sigma v=3 \times 10^{-26} \mathrm{~cm}^{3} / \mathrm{s}$ (the value estimated for a typical thermal relic) to either $b \bar{b}$ or $\tau^{+} \tau^{-}$. The left and right frames show the flux predicted from within the inner $0.5^{\circ}$ and $3^{\circ}$ around the Galactic center, respectively. The error bars shown correspond to the measurements of FGST over its first 14 months of observation (as shown in Ref. [8]). Each curve has been corrected to account for leakage due to FGST's finite point spread function (see Ref. [8] for details).

While the fluxes shown in Fig. 6 do not exceed those measured by FGST, they are quite close-within a factor of $2-3$ below $1-2 \mathrm{GeV}$. As more data are accumulated, and as the astrophysical backgrounds in this region become in- creasingly understood, FGST will likely become sensitive to $5-10 \mathrm{GeV}$ dark matter particles with annihilation cross sections near that predicted for a simple thermal relic. The lone caveat to this conclusion is the inner galaxy does not contain a significant cusp (such as that described by the NFW model), then the gamma ray fluxes from dark matter annihilations could be considerably suppressed.

Recently, the FGST Collaboration has reported its first limits on the gamma ray flux from a number of dwarf spheroidal galaxies, and used this information to constrain the properties of dark matter [59]. Although results are not presented for dark matter particles as light as $5-10 \mathrm{GeV}$ (except in the special case of annihilations to $\mu^{+} \mu^{-}$), the FGST Collaboration found that dark matter particles with a $30 \mathrm{GeV}$ mass and which annihilate to $b \bar{b}$ are restricted to $\sigma|v| \lesssim 6 \times 10^{-26} \mathrm{~cm}^{3} / \mathrm{s}$, assuming an NFW profile shape. Furthermore, the annihilation products of 5$10 \mathrm{GeV}$ dark matter particles are predicted to contribute to the diffuse isotropic gamma ray flux at a level near the current sensitivity of FGST [60].

\section{Indirect detection with charged cosmic rays}

In contrast to most other astrophysical processes, dark matter annihilations are generally predicted to produce equal fluxes of matter and antimatter. An excess of antimatter in the cosmic ray spectrum relative to the predictions of astrophysical models could thus potentially constitute a signal of dark matter annihilations taking place in the halo of the Milky Way.

Once injected into the interstellar medium, cosmic rays undergo a number of processes which can alter their spectra as observed at Earth (for a review, see Ref. [61]). Such processes include deflection by and diffusion through the galactic magnetic field, diffusive reacceleration, convection, and various energy loss processes resulting from
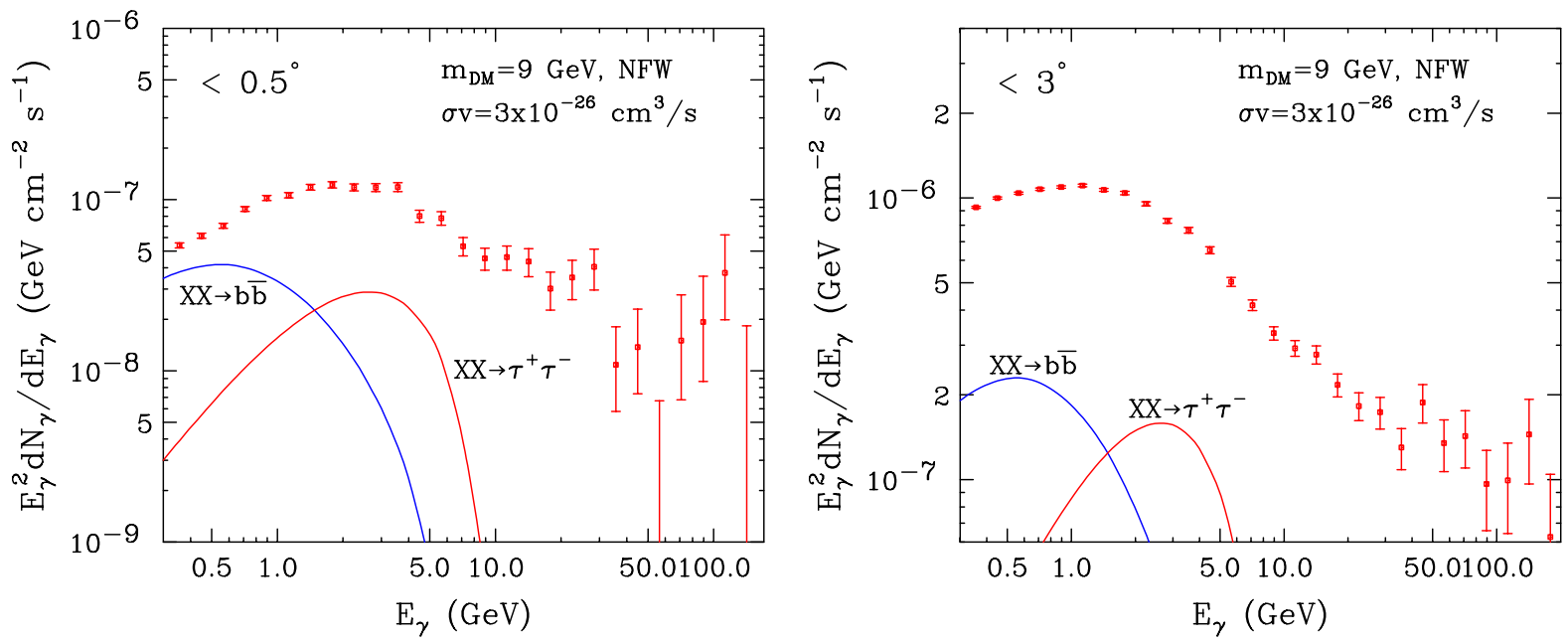

FIG. 6 (color online). The spectrum of gamma rays from $9 \mathrm{GeV}$ dark matter particles annihilating in the inner Milky Way, compared to the measurements of the Fermi Gamma Ray Space Telescope (FGST). Here, we have assumed a dark matter distribution that follows an NFW profile, and an annihilation cross section of $\sigma v=3 \times 10^{-26} \mathrm{~cm}^{3} / \mathrm{s}$. See text for more details. 
interactions with gas, radiation fields, and magnetic fields. To confidently interpret the measurements of the cosmic ray spectrum, these processes must be understood and adequately modeled.

At relatively high energies $(E \geqslant 10 \mathrm{GeV})$, many of these processes play only a relatively minor role. In particular, the effects of convection and reacceleration impact the cosmic ray spectrum much more strongly at low energies. This enables one to interpret the high energy measurements of PAMELA and (in the future) AMS-02 without introducing intractable uncertainties associated with the cosmic ray propagation model. For dark matter with a mass in the $\sim 10 \mathrm{GeV}$ range, however, the resulting annihilation products will be of sufficiently low energy that they will likely be significantly affected by convection and reacceleration, as well as by interactions with the Solar System's magnetic field (i.e. solar modulation). Such effects may make it difficult to interpret these measurements within the context of indirect dark matter searches. That being said, lighter dark matter particles annihilate more often than heavier particles, leading to a greater flux of annihilation products to potentially detect $[46,62]$. Without exploring these competing factors further, we simply comment that light dark matter particles are generally expected to produce large fluxes of antimatter in the $\sim \mathrm{GeV}$ region of the cosmic ray spectrum, but that the complicated nature of cosmic ray propagation over this range of energies may make it difficult to clearly identify such a contribution in existing or future measurements.

\section{Anomalous white dwarf heating}

Dark matter particles with large elastic scattering cross sections (such as those being considered here) can become captured by and annihilate in compact stars at very high rates. In relatively cool stars, such as very old white dwarfs, the energy injected from these annihilations could potentially compete with or exceed the star's luminosity, leading to observable effects $[63,64]$.

As a result of the very high densities of nuclei in white dwarfs, dark matter particles with elastic scattering cross sections of $\sigma \sim 10^{-40} \mathrm{~cm}^{2}$ will scatter numerous times as they pass through the volume of the star. In this optically thick limit, dark matter particles will be gravitationally captured by a white dwarf at a rate given by

$$
\Gamma_{c} \approx\left(\frac{8 \pi}{3}\right)^{1 / 2} \frac{3 G R_{\mathrm{WD}} M_{\mathrm{WD}} \rho_{\mathrm{DM}}}{m_{\mathrm{DM}} \bar{v}},
$$

where $R_{\mathrm{WD}}$ and $M_{\mathrm{WD}}$ are the radius and mass of the white dwarf, respectively, $\rho_{\mathrm{DM}}$ is the dark matter density in the region of the star, and $\bar{v}$ is the dark matter's velocity dispersion in that region. For a typical white dwarf $\left(R_{\mathrm{WD}} \approx\right.$ $0.0083 R_{\odot}, M_{\mathrm{WD}} \approx 0.7 M_{\odot}$ ), this leads to a contribution to the star's luminosity given by [64]

$$
\begin{aligned}
L & \approx \Gamma_{c} m_{\mathrm{DM}} \\
& \approx 2.5 \times 10^{28} \mathrm{GeV} / \mathrm{s}\left(\frac{\rho_{\mathrm{DM}}}{1 \mathrm{GeV} / \mathrm{cm}^{3}}\right)\left(\frac{220 \mathrm{~km} / \mathrm{s}}{\bar{v}}\right) \\
& \approx 4 \times 10^{25} \mathrm{erg} / \mathrm{s}\left(\frac{\rho_{\mathrm{DM}}}{1 \mathrm{GeV} / \mathrm{cm}^{3}}\right)\left(\frac{220 \mathrm{~km} / \mathrm{s}}{\bar{v}}\right) .
\end{aligned}
$$

For comparison, the Stefan-Boltzmann law predicts the luminosity of a blackbody to be

$$
\begin{aligned}
L_{\mathrm{BB}} & =4 \pi R_{\mathrm{WH}}^{2} \sigma T_{\mathrm{WD}}^{4} \\
& \approx 1.9 \times 10^{28} \mathrm{erg} / \mathrm{s}\left(\frac{T_{\mathrm{WD}}}{3000 \mathrm{~K}}\right)^{4}\left(\frac{R_{\mathrm{WD}}}{0.0083 R_{\odot}}\right)^{2} .
\end{aligned}
$$

Comparing Eqs. (42) and (43), we see that a very old ( $T \sim$ $3000 \mathrm{~K}$ ) white dwarf in a typical region of the galactic disk will have only on the order of $0.1 \%$ of its luminosity generated by dark matter annihilations. In regions of high dark matter density and low dark matter velocity dispersion, however, dark matter annihilations can play a considerably greater role. The dark matter density and velocity dispersions within the inner $\sim 10-20$ parsecs of dwarf spheroidal galaxies are typically on the order of $\rho_{\mathrm{DM}} \sim$ $10^{2} \mathrm{GeV} / \mathrm{cm}^{3}$ and $\bar{v} \sim 10 \mathrm{~km} / \mathrm{s}$, respectively [65]. In such an environment, dark matter of the type required to produce the excesses observed by CoGeNT and DAMA will prevent white dwarf stars from cooling below $\sim 5000 \mathrm{~K}$ [64]. This provides an opportunity for future telescopes not only to confirm a dark matter interpretation of the CoGeNT result, but also to potentially map the density and velocity dispersion of dark matter in regions such as dwarf spheroidal galaxies.

\section{CONCLUSIONS}

In this paper, we have discussed the recent excess of low energy events reported by the CoGeNT Collaboration, and considered the possibility that these events, as well as the annual modulation observed by DAMA, are the result of a light $\left(m_{\mathrm{DM}} \sim 5-10 \mathrm{GeV}\right)$ dark matter particle. We find that a common dark matter interpretation may be compatible with the CoGeNT and DAMA signals, as well as the modest excess recently reported by CDMS. We find that this interpretation is also consistent with the null results of XENON10 and the CDMS silicon analysis.

Dark matter with the properties (mass and elastic scattering cross section) required to produce the CoGeNT and DAMA excesses can appear within a variety of theoretical frameworks. From a model independent perspective, we note that a $\sim 5-10 \mathrm{GeV}$ scalar dark matter particle with approximately universal scalar couplings to standard model fermions will naturally produce approximately the observed event rates if its couplings are fixed to obtain the desired thermal relic abundance of dark matter. Fermionic dark matter particles are also a viable possibility, although multiple types of interactions appear necessary if such a 
particle is also to satisfy relic density constraints. Within the context of specific theoretical models, we have discussed both asymmetric dark matter models and neutralinos within extended supersymmetric models, such as the NMSSM. Asymmetric dark matter is especially attractive as an explanation for the CoGeNT and DAMA signals, as in such scenarios the dark matter's mass is related to the proton's mass and is generally predicted to be roughly in the range of $m_{\mathrm{DM}} \sim 1-10 \mathrm{GeV}$.

We have also discussed the implications of the CoGeNT and DAMA excesses for other dark matter search strategies, and find that the prospects for indirect detection are very favorable in many such scenarios. In particular, existing limits from low-threshold neutrino detectors, such Super-Kamiokande, already constrain the dominant annihilation channels of such a WIMP. Searches for dark matter annihilation products with the Fermi Gamma Ray Space
Telescope currently have a level of sensitivity that is very close to being able to test dark matter in a form of a $\sim 5-10 \mathrm{GeV}$ thermal relic.

\section{ACKNOWLEDGMENTS}

We would like to thank Juan Collar for his assistance in understanding the details of the recent CoGeNT result. We also thank B. Feldstein, A. Pierce, and P. Sorensen for helpful discussions. D.H. is supported by the U.S. Department of Energy, including Grant No. DE-FG0295ER40896, and by NASA Grant No. NAG5-10842. A.L.F. and K. M.Z. thank the KITP for their hospitality at the workshop "Direct, Indirect and Collider Signals of Dark Matter," where parts of this work were initiated. A. L.F. is supported by DOE Grant No. DE-FG02-01ER40676 and NSF CAREER Grant No. PHY-0645456.
[1] R. Bernabei et al., Eur. Phys. J. C 67, 39 (2010).

[2] Z. Ahmed et al. (The CDMS-II Collaboration), arXiv:0912.3592.

[3] C.E. Aalseth et al. (The CoGeNT Collaboration), arXiv: 1002.4703 .

[4] O. Adriani et al. (PAMELA Collaboration), Nature (London) 458, 607 (2009).

[5] A. A. Abdo et al. (The Fermi LAT Collaboration), Phys. Rev. Lett. 102, 181101 (2009).

[6] D.P. Finkbeiner, arXiv:astro-ph/0409027; D. Hooper, D. P. Finkbeiner, and G. Dobler, Phys. Rev. D 76, 083012 (2007); G. Dobler and D.P. Finkbeiner, Astrophys. J. 680, 1222 (2008).

[7] G. Dobler, D. P. Finkbeiner, I. Cholis, T. R. Slatyer, and N. Weiner, arXiv:0910.4583.

[8] L. Goodenough and D. Hooper, arXiv:0910.2998.

[9] D. Hooper, P. Blasi, and P.D. Serpico, J. Cosmol. Astropart. Phys. 01 (2009) 025; H. Yuksel, M.D. Kistler, and T. Stanev, Phys. Rev. Lett. 103, 051101 (2009); S. Profumo, arXiv:0812.4457; F. A. Aharonian, A. M. Atoyan, and H. J. Volk, Astron. Astrophys. 294, L41 (1995); I. Buesching, O.C. de Jager, M.S. Potgieter, and C. Venter, arXiv:0804.0220; L. Zhang and K. S. Cheng, Astron. Astrophys. 368, 1063 (2001); M. Kaplinghat, D. J. Phalen, and K. M. Zurek, J. Cosmol. Astropart. Phys. 12 (2009) 010; J.P. Harding and K. N. Abazajian, Phys. Rev. D 81, 023505 (2010); C. M. Kelso and D. Hooper, arXiv:1001.1749.

[10] K. M. Zurek, Phys. Rev. D 79, 115002 (2009); I. Cholis and N. Weiner, arXiv:0911.4954.

[11] J. Angle et al. (XENON10 Collaboration), Phys. Rev. D 80, 115005 (2009)

[12] D. B. Kaplan, Phys. Rev. Lett. 68, 741 (1992); S. Nussinov, Phys. Lett. 165B, 55 (1985); S. M. Barr, Phys. Rev. D 44, 3062 (1991); S. M. Barr, R. S. Chivukula, and E. Farhi, Phys. Lett. B 241, 387 (1990); S. B. Gudnason,
C. Kouvaris, and F. Sannino, Phys. Rev. D 73, 115003 (2006); S. Dodelson, B. R. Greene, and L. M. Widrow, Nucl. Phys. B372, 467 (1992); M. Fujii and T. Yanagida, Phys. Lett. B 542, 80 (2002); R. Kitano and I. Low, Phys. Rev. D 71, 023510 (2005); R. Kitano, H. Murayama, and M. Ratz, Phys. Lett. B 669, 145 (2008); G. R. Farrar and G. Zaharijas, Phys. Rev. Lett. 96, 041302 (2006); Z. Berezhiani and A. Lepidi, Phys. Lett. B 681, 276 (2009); G. D. Kribs, T.S. Roy, J. Terning, and K. M. Zurek, Phys. Rev. D 81, 095001 (2010); Y. Cai, M. A. Luty, and D. E. Kaplan, arXiv:0909.5499; H. An, S.L. Chen, R. N. Mohapatra, and Y. Zhang, J. High Energy Phys. 03 (2010) 124.

[13] D. E. Kaplan, M. A. Luty, and K. M. Zurek, Phys. Rev. D 79, 115016 (2009); T. Cohen and K. M. Zurek, Phys. Rev. Lett. 104, 101301 (2010).

[14] J. Angle et al. (XENON Collaboration), Phys. Rev. Lett. 100, 021303 (2008).

[15] D. S. Akerib et al. (CDMS Collaboration), Phys. Rev. Lett. 96, 011302 (2006).

[16] J. Filippino (CDMS Collaboration), Nuovo Cimento Soc. Ital. Fis. C 32, 45 (2009).

[17] F. Petriello and K. M. Zurek, J. High Energy Phys. 09 (2008) 047.

[18] S. Chang, A. Pierce, and N. Weiner, Phys. Rev. D 79, 115011 (2009).

[19] M. Fairbairn and T. Schwetz, J. Cosmol. Astropart. Phys. 01 (2009) 037.

[20] C. Savage, K. Freese, P. Gondolo, and D. Spolyar, J. Cosmol. Astropart. Phys. 09 (2009) 036; C. Savage, G. Gelmini, P. Gondolo, and K. Freese, J. Cosmol. Astropart. Phys. 04 (2009) 010.

[21] E. M. Drobyshevski, Mod. Phys. Lett. A 23, 3077 (2008).

[22] A. K. Drukier, K. Freese, and D. N. Spergel, Phys. Rev. D 33, 3495 (1986).

[23] C. S. Kochanek, Astrophys. J. 457, 228 (1996). 
[24] G. Jungman, M. Kamionkowski, and K. Griest, Phys. Rep. 267, 195 (1996).

[25] G. Duda, A. Kemper, and P. Gondolo, J. Cosmol. Astropart. Phys. 04 (2007) 012.

[26] G. Fricke, C. Bernhardt, K. Heilig, L. A. Schaller, L. Schellenberg, E. B. Shera, and C. W. de Jager, At. Data Nucl. Data Tables 60, 177 (1995).

[27] S. Yellin, Phys. Rev. D 66, 032005 (2002).

[28] R. Bernabei et al., Eur. Phys. J. C 53, 205 (2008).

[29] R. Foot, arXiv:0804.4518.

[30] J. Graichen, K. Maier, J. Schuth, A. Siepe, and W. von Witsch, Nucl. Instrum. Methods Phys. Res., Sect. A 485, 774 (2002); 501, 630(E) (2003).

[31] J. Kopp, T. Schwetz, and J. Zupan, J. Cosmol. Astropart. Phys. 02 (2010) 014.

[32] P. Sorenson, Ph.D. thesis, Brown University, 2008.

[33] A. Manzur, A. Curioni, L. Kastens, D. N. McKinsey, K. Ni, and T. Wongjirad, Phys. Rev. C 81, 025808 (2010).

[34] C.P. Burgess, M. Pospelov, and T. ter Veldhuis, Nucl. Phys. B619, 709 (2001); S. Andreas, T. Hambye, and M.H. G. Tytgat, J. Cosmol. Astropart. Phys. 10 (2008) 034; Y. G. Kim and S. Shin, J. High Energy Phys. 05 (2009) 036.

[35] R. Foot, Int. J. Mod. Phys. D 13, 2161 (2004); Phys. Rev. D 78, 043529 (2008).

[36] J.L. Feng and J. Kumar, Phys. Rev. Lett. 101, 231301 (2008); J. L. Feng, J. Kumar, and L. E. Strigari, Phys. Lett. B 670, 37 (2008).

[37] J. Giedt, A. W. Thomas, and R. D. Young, Phys. Rev. Lett. 103, 201802 (2009).

[38] R. D. Young and A. W. Thomas, Phys. Rev. D 81, 014503 (2010); D. Toussaint and W. Freeman (MILC Collaboration), Phys. Rev. Lett. 103, 122002 (2009).

[39] M. Beltran, D. Hooper, E. W. Kolb, and Z. C. Krusberg, Phys. Rev. D 80, 043509 (2009).

[40] See, e.g., A. Bottino, N. Fornengo, and S. Scopel, Phys. Rev. D 67, 063519 (2003); A. Bottino, F. Donato, N. Fornengo, and S. Scopel, Phys. Rev. D 78, 083520 (2008); V. Niro, A. Bottino, N. Fornengo, and S. Scopel, Phys. Rev. D 80, 095019 (2009); A. Bottino, F. Donato, N. Fornengo, and S. Scopel, Phys. Rev. D 72, 083521 (2005).

[41] A. Bottino, F. Donato, N. Fornengo, and S. Scopel, Phys. Rev. D 68, 043506 (2003).

[42] C. Amsler et al. (Particle Data Group), Phys. Lett. B 667, 1 (2008).

[43] E. Kuflik, A. Pierce, and K. M. Zurek, arXiv:1003.0682; D. Feldman, Z. Liu, and P. Nath, arXiv:1003.0437.

[44] D. Hooper and T. Plehn, Phys. Lett. B 562, 18 (2003).
[45] J. F. Gunion, D. Hooper, and B. McElrath, Phys. Rev. D 73, 015011 (2006).

[46] F. Ferrer, L. M. Krauss, and S. Profumo, Phys. Rev. D 74, 115007 (2006).

[47] A. Gould, Astrophys. J. 388, 338 (1992).

[48] A. Gould, Astrophys. J. 321, 560 (1987).

[49] K. Griest and D. Seckel, Nucl. Phys. B283, 681 (1987); B296, 1034(E) (1988).

[50] A. Gould, Astrophys. J. 321, 571 (1987).

[51] S. Desai et al. (Super-Kamiokande Collaboration), Phys. Rev. D 70, 083523 (2004); 70, 109901(E) (2004).

[52] J. L. Feng, J. Kumar, J. Learned, and L. E. Strigari, J. Cosmol. Astropart. Phys. 01 (2009) 032.

[53] D. Hooper, F. Petriello, K. M. Zurek, and M. Kamionkowski, Phys. Rev. D 79, 015010 (2009).

[54] J. Edsjo (private communication).

[55] P. Gondolo, J. Edsjo, P. Ullio, L. Bergstrom, M. Schelke, and E. A. Baltz, J. Cosmol. Astropart. Phys. 07 (2004) 008.

[56] L. Bergstrom, P. Ullio, and J. H. Buckley, Astropart. Phys. 9, 137 (1998); V. Berezinsky, A. Bottino, and G. Mignola, Phys. Lett. B 325, 136 (1994).

[57] N. W. Evans, F. Ferrer, and S. Sarkar, Phys. Rev. D 69, 123501 (2004); L. Bergstrom and D. Hooper, Phys. Rev. D 73, 063510 (2006); G. D. Martinez, J.S. Bullock, M. Kaplinghat, L. E. Strigari, and R. Trotta, J. Cosmol. Astropart. Phys. 06 (2009) 014.

[58] J. F. Navarro, C. S. Frenk, and S. D. M. White, Astrophys. J. 462, 563 (1996); J. F. Navarro, C. S. Frenk, and S. D. M. White, Astrophys. J. 490, 493 (1997).

[59] A. A. Abdo et al., Astrophys. J. 712, 147 (2010).

[60] K. N. Abazajian, P. Agrawal, Z. Chacko, and C. Kilic, arXiv:1002.3820.

[61] A. W. Strong, I. V. Moskalenko, and V. S. Ptuskin, Annu. Rev. Nucl. Part. Sci. 57, 285 (2007)

[62] A. Bottino, F. Donato, N. Fornengo, and S. Scopel, Phys. Rev. D 77, 015002 (2008); A. Bottino, F. Donato, N. Fornengo, and P. Salati, Phys. Rev. D 72, 083518 (2005).

[63] I. V. Moskalenko and L. L. Wai, Astrophys. J. 659, L29 (2007); G. Bertone and M. Fairbairn, Phys. Rev. D 77, 043515 (2008); M. McCullough and M. Fairbairn, Phys. Rev. D 81, 083520 (2010).

[64] D. Hooper, D. Spolyar, A. Vallinotto, and N. Y. Gnedin, Phys. Rev. D 81, 103531 (2010).

[65] M. G. Walker, M. Mateo, E. W. Olszewski, O. Y. Gnedin, X. Wang, B. Sen, and M. Woodroofe, arXiv:0708.0010.

[66] Z. Ahmed et al. (CDMS Collaboration), Phys. Rev. Lett. 102, 011301 (2009). 